\title{
Comparison of different double immunostaining protocols for paraffin embedded liver tissue
}

\author{
Alexander Schütz, Andrea Tannapfel and \\ Christian Wittekind * \\ Institute of Pathology, University of Leipzig, Germany
}

Received 6 July 1998

Accepted 2 July 1999

Most of the double immunostaining protocols that have been introduced so far have been developed for application on fresh frozen material or based on different species antibodies.

In liver tissue, general problems of double immunostaining techniques are further complicated by tissue-specific difficulties, such as necrosis or high intracellular protein content.

To assess a reliable double immunostaining protocol for archived, paraffin embedded liver tissue, different protocols based on the use of same species primary antibodies were evaluated in terms of sensitivity, specificity and non-specific background staining in pathological liver specimens.

We compared peroxidase-anti-peroxidase, alkaline phosphatase-anti-alkaline phosphatase (PAP/APAP), labelledavidin-biotin (LAB/LAB) and digoxigenin-anti-digoxigenin (dig-a-dig/PAP) techniques using different cytokeratin antibodies and an antibody against PCNA.

Comparison of the double immunostaining techniques revealed a high sensitivity and specificity in all procedures. Sections, which were stained employing PAP/APAPtechnique, displayed a higher background staining compared to sections which were treated with the $\mathrm{LAB} / \mathrm{LAB}$ or dig-adig/PAP protocol. In contrast to the dig-a-dig/PAP protocol, the $\mathrm{LAB} / \mathrm{LAB}$ technique provides a better time/cost relationship.

Therefore, we would like to recommend a modified $\mathrm{LAB} / \mathrm{LAB}$ protocol for simultaneous detection of different antigens in archived liver tissue.

Keywords: Double immunohistochemistry, archived liver tissue

${ }^{*}$ Correspondence to: Prof. Dr. med. Christian Wittekind, Institute of Pathology, University of Leipzig, Liebigstr. 26, D-04103 Leipzig, Germany. Tel.: +49 341 9715000; Fax: +49 3419715009.

\section{Introduction}

Several immunohistochemical protocols are routinely applicable in fixed, paraffin embedded material. To demonstrate the distribution of different antigens simultaneously, only a few double immunolabelling protocols have been published so far.

In archived liver tissue, many efforts have been attempted to establish a double immunostaining protocol which gives reproducible and reliable staining results. All these protocols are only applicable on fresh frozen liver tissue [6] or employ different species antibodies [3,7,11,19,21].

For this reason we tested diverse double-immunostaining protocols using primary antibodies of the same species on routinely available fixed liver tissue from different pathological conditions.

Common problems of single immunostaining, for instance, the loss of specificity and sensitivity due to fixation derived antigen loss and high non-specific background staining, are amplified in double immunostaining procedures $[1,3,19]$. In liver tissue these problems are accompanied by tissue specific difficulties, such as high endogenous intracellular protein or biotin concentration and necrosis occurring at the time interval between tissue removal and fixation [22]. Furthermore, pathological features such as cholestasis cause problems in immunohistochemical procedures.

We employed double immunostaining protocols based on the following techniques: (a) labelled avidinbiotin technique in the first and second step of immunostaining (LAB/LAB) [22]; (b) peroxidase-antiperoxidase technique in the first step, and alkaline phosphatase-anti-alkaline phosphatase technique in the second step (PAP/APAP) $[1,10]$; and (c) digoxigenin-anti-digoxigenin technique combined with peroxidase-anti-peroxidase technique (dig-a-dig/PAP) $[10,12]$ in the third.

To test the above mentioned techniques, we used mouse antibodies raised against various cytokeratin 
(CK) epitopes and against proliferation nuclear cell antigen (PCNA). We investigated CK 19 because of its restricted expression within bile duct epithelial cells [4, 25] and PCNA because of its nuclear staining pattern in proliferating cells [7,9]. Since the antigens evaluated in our study are highly unstable and easily destroyed due to tissue processing [7,25], a precise evaluation of the sensitivity of the protocols is necessary. Further, we evaluated all protocols for their time/cost relationship.

\section{Material and methods}

Double-immunoperoxidase staining was performed on resection specimens and fine needle biopsies of cirrhotic human liver tissue (10 cases), focal nodular hyperplasia (10 cases) and normal human liver tissue (4 cases).

Cirrhosis and focal nodular hyperplasia were diagnosed by common histology using haematoxilin-eosin (HE) and elastica van Gieson staining (EvG). The tissue was fixed in $4 \%$ saline buffered formaldehyde, embedded in paraffin and cut into $4 \mu \mathrm{m}$ thick sections.

Subsequently, the sections were deparaffinated and rinsed three times in $0.1 \mathrm{M}$ phosphate-buffered saline, $\mathrm{pH} 7.4$ (PBS). To reduce endogenous peroxidase activity, the sections were treated with $3.0 \% \mathrm{H}_{2} \mathrm{O}_{2}$ in PBS for $20 \mathrm{~min}$.

Following this procedure, the tissue was heated three times in citrate buffer, $\mathrm{pH} 6.0$ (CB) for $5 \mathrm{~min}$ in a microwave oven.

\subsection{Single immunolabelling of serial sections}

First, the labelled avidin-biotin, peroxidase-antiperoxidase, alkaline phosphatase-anti-alkaline phosphatase and digoxigenin-anti-digoxigenin techniques were employed for single immunostaining to detect the distribution of pan-CK, CK 19 and PCNA in serial sections. These techniques were performed in the same way as explained in the following detailed description of the double immunostaining protocols and as published in the literature $[1,8,10,13,22]$.

\subsection{Double labelling using LAB-technique}

After rinsing in PBS, the sections were incubated with avidin (Vector, Burlingame, CA, USA) and biotin (Vector) for $15 \mathrm{~min}$ each to block non-specific binding sites of the detection system. Afterwards, the tissue was treated with PBS containing 5\% normal goat serum (PBS-NGS; DAKO, Carpinteria, CA, USA) to block non-specific binding sites of the secondary antibody.

Then, the sections were incubated with mouse antiPCNA (Dianova, Hamburg, Germany; 1 to 40 in PBS) overnight. After rinsing, the sections were processed using the labelled avidin-biotin-technique (LAB) including the incubation with biotinylated goat-antimouse antibody (BioGenex, San Ramon, CA, USA; 1 to 50 in PBS) and the incubation with peroxidase labelled avidin (BioGenex; 1 to 50 in PBS) for $30 \mathrm{~min}$ each.

PCNA-immunoreactivity was visualised by black staining with nickel-enhanced diaminobenzidine (DAB/Ni; $1.0 \mathrm{mg}$ ammoniumnickelsulphate (Riedel de Häen, Seelze, Netherlands)/1 ml DAB (Sigma, Deisenhofen, Germany)) as a chromogen.

After rinsing the sections three times in PBS, the tissue was processed for $20 \mathrm{~min}$ in $\mathrm{CB}$ as an acid medium. Subsequently, the tissue was reacted with mouse antiCK 19 (Quartett, Berlin, Germany; 1 to 10 in PBS) overnight.

After rinsing the tissue three times in PBS, the LABtechnique was applied again. In the second step of double-immunostaining, avidin-conjugated alkalinephosphatase (BioGenex; 1 to 50 in PBS) as a marker enzyme, and fast Red (BioGenex) as a chromogen were used.

\subsection{Double labelling using peroxidase- anti-peroxidase and alkaline phosphatase-anti-alkaline phosphatase technique}

The treatment of the sections with PBS-NGS was followed by incubation with mouse a-PCNA (Dianova, Hamburg, Germany; 1 to 40 in PBS) overnight. After rinsing in PBS, the sections were reacted with goat-anti-mouse (BioGenex; 1 to 50 in PBS) and mouse peroxidase-anti-peroxidase (BioGenex; 1 to 50 in PBS) for $1 \mathrm{~h}$ each. PCNA-immunoreactivity was visualized using $\mathrm{DAB} / \mathrm{Ni}$ as a chromogen.

After rinsing the sections three times in PBS, the tissue was processed for $20 \mathrm{~min}$ in $\mathrm{CB}$ as an acid medium.

After incubation with PBS-NGS for $20 \mathrm{~min}$, the tissue was then reacted with mouse anti-CK 19 (Quartett, Berlin, Berlin; 1 to 10 in PBS) overnight.

After rinsing the tissue in PBS, the APAP-technique was applied using alkaline phosphatase (BioGenex; 1 to 50 in PBS) as a marker enzyme and fast Red (BioGenex) as a chromogen. 


\subsection{Double labelling using dig-a-dig and PAP technique}

The digoxigenin-labelling of the mouse anti-CK antibody required the isolation of the pure IgG-fraction from the stabilising fetal calf serum. The primary IgG concentration was $220 \mu \mathrm{g} / \mathrm{ml}$. Separation was obtained using the ammoniumsulphate/caprylic acid method, described by McKinney and Parkinson [15]. The resulting $\mathrm{IgG}$ was dissolved in $1 \mathrm{ml} 0.1 \mathrm{M}$ carbonate buffer, $\mathrm{pH}$ 8.5. This solution was reacted with $50 \mu \mathrm{g}$ digoxigenin-3-O-methyl-carbonyl- $\varepsilon$-aminocaproic acid hydroxy-succinimidester (Boehringer, Mannheim, Germany) dissolved in $50 \mu \mathrm{l}$ dimethylformamide (Boehringer) for $1 \mathrm{hr}$ at room temperature and dialysed three times against PBS [10].

The resultant dig-tagged antibody is storable for at least 6 mo at $4^{\circ} \mathrm{C}$.

Immunostaining was carried out as follows: after incubation in PBS-NGS to block non-specific binding sites, the sections were reacted with mouse antiPCNA overnight. After rinsing three times in PBS, the sections were immunostained using the PAP-technique with peroxidase as a marker enzyme and $\mathrm{DAB} / \mathrm{Ni}$ as a chromogen.

For visualisation of cytokeratin-immunoreactivity, the sections were reacted with $\mathrm{H}_{2} \mathrm{O}_{2}$ to abolish remaining activity of the marker enzyme. Following incubation in PBS containing 5\% normal mouse serum (PBSNMS) to block non-specific binding sites, the sections were reacted with the dig-tagged cytokeratin antibody in a dilution of $3 \mu \mathrm{g} / \mathrm{ml}$ in PBS for two days. After rinsing, the tissue was incubated with peroxidase-labelled mouse a-digoxigenin (Boehringer; 1 to 40 in PBS). The immunostaining was carried out using $\mathrm{DAB}$ as a chromogen. The sections were rinsed in Aqua dest., dried and then coverslipped with Aqua-tex (Merck, Darmstadt, Germany).

\subsection{Controls}

Negative controls were obtained by employing normal mouse serum (NMS, DAKO, Carpinteria, CA, USA) instead of primary antibodies. Unspecific staining was not apparent. For positive control of PCNA, colon carcinoma specimens were used, and for pan-CK and CK 19 prostate tissue was employed. Additionally, double immunostaining experiments were supplemented by single immunolabelling based on the above technique.
The final count of PCNA-positive cells was established after examination of the entire slide to avoid overestimation due to focal heterogeneity. On each slide 1000 hepatocytes and 100 bile duct epithelial cells in representative areas were examined for their PCNA-and cytokeratin-expression.

The sensitivity for detecting PCNA, using different double immunostaining procedures, was compared by image analyses. Therefore we estimated the relative positive areas of PCNA-immunoreactivity in comparable fields. We employed a Olympus BH-2 microscope and the Cell Image Retrieval and Evaluation System (Cires 3.0, Kontron Elektronik, München, Germany), from Kontron.

\section{Results}

\subsection{Immunostaining for pan-cytokeratin}

In normal liver tissue all bile duct epithelial cells and hepatocytes exhibited an immunoreactivity for pancytokeratin at the basolateral cell membrane.

In cirrhotic liver the entire count of bile duct epithelial cells as well as all hepatocytes expressed cytokeratin epitopes which were identified by antibodies against pan-cytokeratin.

In focal nodular hyperplasia the cytokeratin expression pattern showed an immunoreactivity of all bile duct epithelial cells and all hepatocytes.

The antigen was located in all sections at the basolateral cell membrane.

The connective tissue within portal tracts showed neither specific nor non-specific immunostaining for pan cytokeratin.

\subsection{Immunostaining for Cytokeratin 19}

In normal liver tissue, immunoreactivity for CK 19 was only visible at the basolateral cell membrane of the bile duct epithelial cells. Hepatocytes were uniformly negative for CK 19.

In cirrhotic liver, CK 19 immunostaining was observed at the basolateral cell membrane of all epithelial cells within pre-existing bile ducts and ductular proliferations.

Some hepatocytes $(5 / 100)$ exhibited a weak immunostaining for CK 19, these cells were scattered around the cirrhotic septa.

In cases of focal nodular hyperplasia, CK 19immunoreactivity was obtained in all bile duct epithe- 
lial cells with very few hepatocytes (2/100) exhibiting weak immunostaining for CK 19 in areas adjacent to fibrotic septa.

No staining for CK 19 was detectable in the connective tissue cells within portal tracts.

\subsection{Immunostaining for PCNA}

In normal liver tissue, very few bile duct epithelial cells $(5 / 100)$ exhibited positive nuclear staining for PCNA. In contrast, a high number of hepatocytes (20/100) were immunostained for PCNA.

In cirrhosis, very few epithelial cells in pre-existing bile ducts (10/100) were PCNA-positive, whereas in ductular proliferations a higher number of PCNAexpressing cells $(20 / 100)$ were observed. Within the nodules of regeneration, a high number of hepatocytes (50/100) exhibited a PCNA-immunoreactivity.

In focal nodular hyperplasia, very few bile duct epithelial cells $(15 / 100)$ were immunostained for PCNA, whereas many hepatocytes $(40 / 100)$ exhibited a nuclear staining for this antigen.

In all cases, PCNA-immunostained fibroblasts were occasionally demonstrable within the fibrotic septa.

\subsection{Double immunostaining}

In normal liver, CK 19-immunoreactivity was restricted to bile duct epithelial cells, whereas PCNA was detectable in very few bile duct epithelial cells and in approximately $20 \%$ of the hepatocytes. Bile duct epithelial cells, which showed nuclear staining for PCNA were, without exception, double immunostained for PCNA and CK 19 (Fig. 1a, b).

In cirrhosis, almost all bile duct epithelial cells and a few hepatocytes close to fibrous septa were immunostained for CK 19. Bile duct epithelial cells, which were PCNA-positive were double immunostained for PCNA and for CK 19. Further, some of the hepatocytes which were immunostained for CK 19 exhibited a double immunoreactivity for CK 19 and PCNA (Fig. 2).

In $F N H$, all bile duct epithelial cells and very few hepatocytes showed an immunoreactivity for CK 19. Some bile duct epithelial cells and numerous hepatocytes exhibited an immunostaining for PCNA. All bile duct epithelial cells, which were immunostained for PCNA and some of the hepatocytes that were immunostained for CK 19, showed a double immunostaining.

\subsection{Comparison of single and double immunostaining results}

All double immunostaining protocols compared here exhibited nearly identical results in terms of sensitivity and specificity for detecting PCNA and CK 19.

The evaluation of the relative positive areas in comparable fields as a standard of comparison in image analyses, verified the similarity of the protocols in terms of their sensitivity, as well. The mean value of relative positive areas when was 20.52 when employing the LAB/LAB-technique, 23.19 when using the PAP/APAP-protocol and 26.32 when employing the dig-a-dig/PAP protocol (Fig. 3).

The sections which were double-immunostained employing the PAP/APAP protocol generally showed higher non-specific background staining. This nonspecific background staining was predominantly observed in the cytoplasm of hepatocytes and within necrotic and cholestatic areas.

In contrast, in serial sections immunostained with the LAB/LAB or the dig-a-dig/PAP protocols, a very low non-specific background staining was observed.

\section{Discussion}

Since immunohistochemistry of liver tissue is accompanied by specific problems [22], no reliable double immunostaining protocol employing primary antibodies of the same species was available for routinely fixed liver tissue up to now $[3,6,16]$.

We investigated a LAB/LAB, a PAP/APAP and a dig-a-dig/PAP protocol to recommend a reliable double immunostaining technique for archived liver tissue. All protocols presented here showed a similar sensitivity that was verified by cell count and image analyses in comparable fields (Fig. 3). Our data indicated that the PAP/APAP protocol is not an appropriate double immunostaining protocol for archived liver tissue because of conspicuous non-specific background staining.

Since the intensity of staining is dependent on the peroxidase activity, the LAB/LAB protocol allows a lower concentration of the primary antibody and shorter staining procedures which reduces the nonspecific background staining $[8,13]$.

Applying LAB/LAB and dig-a-dig/PAP-techniques, excellent results in terms of non-specific background staining were obtained (Fig. 1a, b). The preparation of dig-tagged antibodies as described by Härtig et al. [10] circumvents a possible loss of specificity due to the 
(a)

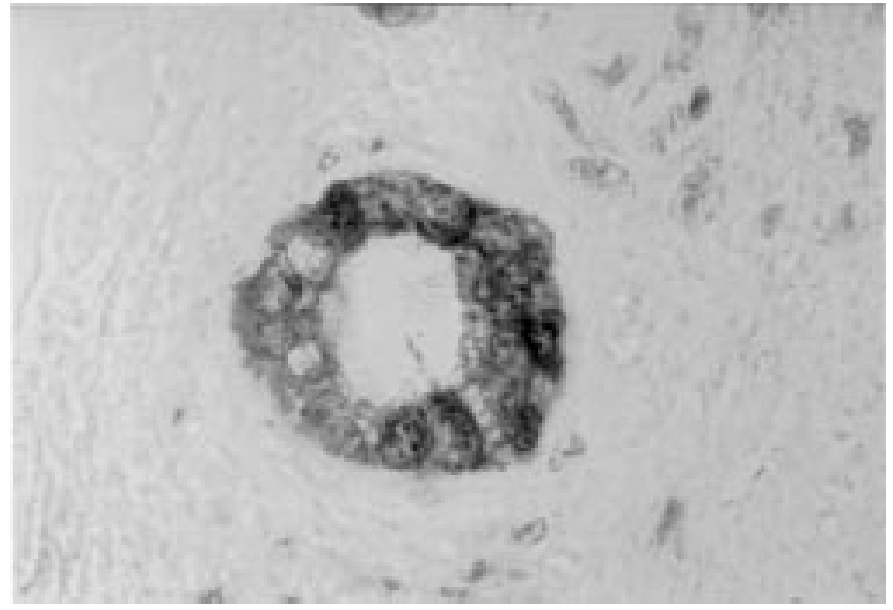

(b)

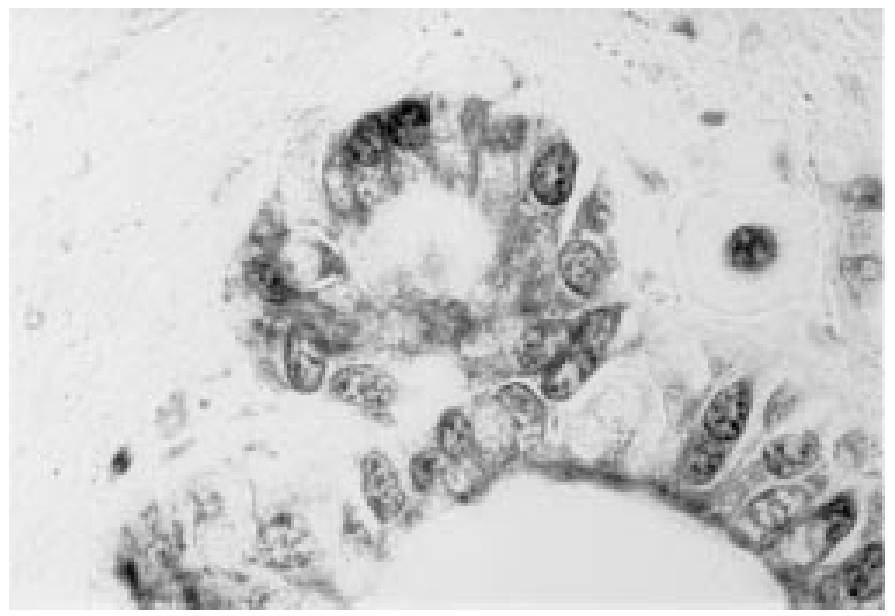

Fig. 1. (a) Bile duct epithelial cells double immunostained using LAB/LAB technique (CK 19: red, PCNA: black; original magnification 100-fold). (b) Bile duct epithelial cells double immunostained using dig-a-dig/PAP method (CK 19: brown, PCNA: black; original magnification 100-fold).

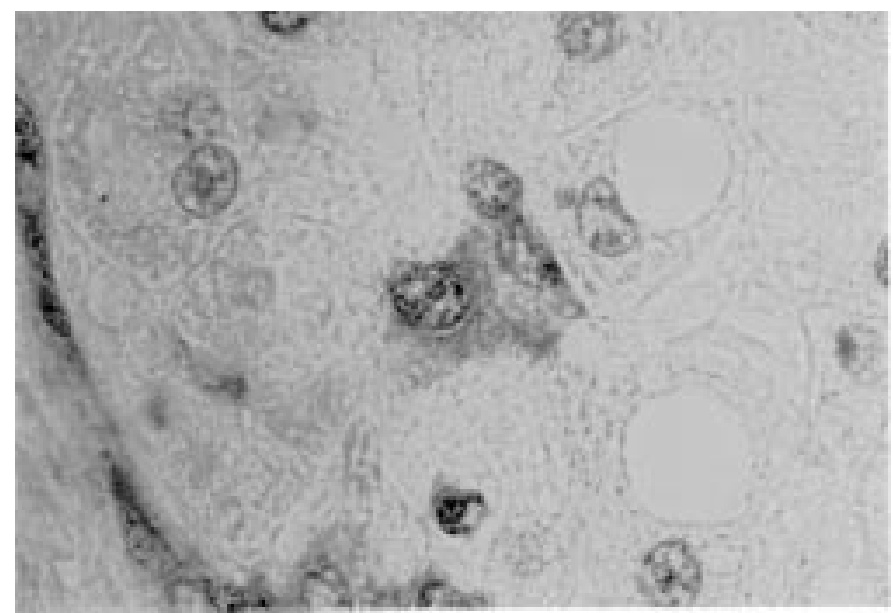

Fig. 2. Hepatocyte expressing CK 19 (red) and PCNA (black) (original magnification 100-fold). 


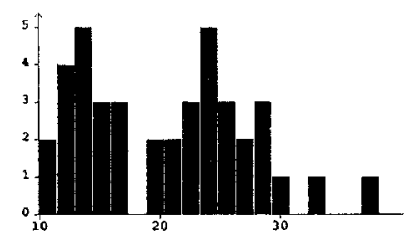

(a)

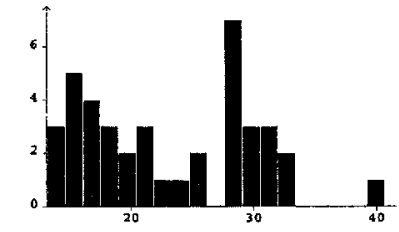

(b)

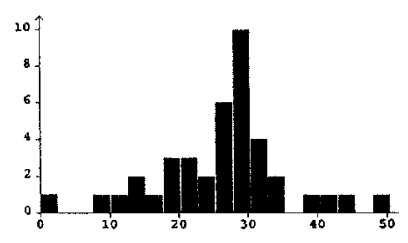

(c)

Fig. 3. Value of relative positive areas for detection of PCNA using LAB/LAB (a), PAP/APAP (b) and dig-a-dig/PAP (c) protocol

binding of the second step detection system to remnant first step antibodies $[10,12]$. The disadvantage of this dig-a-dig-technique is the length of time (about 3-fold) and material due to the tagging procedure.

Therefore, we developed a modified LAB/LABtechnique. The binding of second step detection system to remnant first step primary antibodies was omitted by a prolonged acid buffer rinsing after the first step of immunolabelling. The introduction of an additional treatment in an acidic medium causes a dissociation of remaining immune reagents and their peroxidase labels.

Our results showed that in cirrhosis and in FNH, a large amount of double immunostained bile duct epithelial cells and hepatocytes are detectable. This supports the hypotheses that assume an origin of ductular proliferations from proliferated pre-existing bile ducts $[14,17,20]$ and from transdifferated hepatocytes $[2,5,14,23,24]$.

We could demonstrate that even in routinely fixed, archived liver tissue a double-immunostaining procedure using primary antibodies of the same species gives reproducible staining results when a modified $\mathrm{LAB} / \mathrm{LAB}$ protocol is used. This method is recommendable because of its high specificity and sensitivity, and its very low non-specific background staining. In addition, the time/cost relationship is acceptable.

\section{Acknowledgements}

We wish to thank Dr W. Härtig for supporting the digoxigenin-tagging of the primary antibody against pan-cytokeratin, and Mrs M. Fuegenschuh for excellent technical assistance.

\section{References}

[1] G. Brückner, A. Schütz, W. Härtig, K. Brauer, B.R. Paulke and V. Bigl, Projection of non-cholinergic basal forebrain neurons ensheathed with perineuronal nets to rat mesocortex, J. Chem. Neuroanat. 8 (1994), 14-18.
[2] M.M. Butron Vila, J. Hart and V.J. Desmet, Cholestatic features in focal nodular hyperplasia of the liver, Liver 4 (1984), 387395.

[3] C.M. Chu, C.T. Yeh, I.S. Sheen and Y.F. Liaw, Subcellular localization of hepatitis B core antigen in relation to hepatocyte regeneration in chronic hepatitis B, Gastroenterology 109 (1995), 1926-1932.

[4] V.J. Desmet, Congenital disease of intrahepatic bile ducts: Variations on the theme "ductul plate malformation", J. Hepatol. 16 (1992), 1069-1083.

[5] V.J. Desmet, Modulation of biliary epithelium, in: Modulation of Liver Cell Expression, H. Popper, I.M. Arias, P.C. Heinrich, D. Keppler and L. Landmann, eds, MTP, Lancaster, 1987, pp. 195-214.

[6] L. Garcia-Buey, M. Lopez-Botet, A. Garcia-Sanchez, M.A. Balbao, J. Aramburu, C. Garcia-Monzon, A. Acevedo and R. Moreno-Otero, Variability in the expression of a beta 2-microglobulin epitope on hepatocytes in chronic type C hepatitis on treatment with interferon, Hepatology 17 (1993), 372-382.

[7] C. Garcia-Monzon, R. Moreno-Otero, J.M. Pajares, A. GarciaSanchez, M. Lopez-Botet, M.O. de Landazuri and F. SanchezMadrid, Expression of a novel antigen on intrahepatic CD8+ T lymphocytes in viral chronic active hepatitis, Gastroenterology 98 (1990), 1029-1035.

[8] J.L. Guesdon, T. Ternynck and S. Avrameas, The use of avidinbiotin interaction in immunoenzymtic techniques, J. Histochem. Cytochem. 27 (1979), 1131-1139.

[9] P.A. Hall and A.L. Woods, Immunohistochemical markers of cellular proliferation: achievments, problems and prospects, Cell. Tissue Kinet. 23 (1990), 505-522.

[10] W. Härtig, D. Hausen, K. Brauer, T. Arendt, V. Bigl and G. Brückner, Digoxigenin-tagged anti GFAP and multiple labelling of human glia, vessels and $\beta$-amyloid, NeuroReport 5 (1994), 573-576.

[11] Y. Haruna, K. Saito, S. Spaulding, M.A. Nalesnik and M.A. Gerber, Identification of bipotential progenitor cells in human liver development, Hepatology 23 (1996), 476-481.

[12] H.J. Höltke, W. Ankenbauer, K. Mühlegger, P. Rein, G. Sagner, R. Seibl and T. Walter, The didoxigenin (DIG) system for non-radioactive labelling and detection of nucleic acids - an overview, Cell. Mol. Biol. 41 (1995), 883-905.

[13] S.M. Hsu, L. Raine and H. Fanger, Use of avidin-biotinperoxidase complex $(\mathrm{ABC})$ in immunoperoxidase techniques: A comparison between $\mathrm{ABC}$ and unlabelled antibody (PAP) procedures, J. Histochem. Cytochem. 29 (1981), 577-580. 
[14] O. Klinge, Intrahepatische Cholangitiden auf vermutlich immunologischer Basis, Verh. Dtsch. Ges. Path. 79 (1995), 198232.

[15] M.M. McKinney and A. Parkinson, A simple, nonchromatographic procedure to purify immunoglobulins from serum and ascites fluid, J. Immunol. Methods 96 (1987), 271-278.

[16] G.F. Morris and M.B. Matthews, Regulation of proliferating cell nuclear antigen during the cell cycle, J. Biol. Chem. 264 (1989), 13 856-13864.

[17] Y. Nakanuma and G. Ohta, Immunohistochemical study on bile ductular proliferation in various hepatobiliary diseases, Liver 6 (1986), 205-211.

[18] J. Rudi, R. Waldherr, R. Raedsch and B. Kommerell, Hepatocyte proliferation in primary biliary cirrhosis as assessed by proliferating cell nuclear antigen and Ki-67 antigen labelling, J. Hepatol. 22 (1995), 43-49.

[19] N.G. Ryley, A.R. Heryet, R. Goldin, J. Monjardino, J. Saldanha and K.O. Fleming, Co-expression of markers for hepatitis delta and hepatitis B viruses in human liver, Histopathology 20 (1992), 331-337.
[20] P.A. Slott, M.H. Liu and N. Tavoloni, Origin, pattern, and mechanism of bile duct proliferation following biliary obstruction in the rat, Gastroenterology 99 (1990), 466-477.

[21] Y. Tanaka, N. Funaki, K.M. Mak, C. Kim and C.S. Liebers, Effects of ethanol and vitamin A on proliferation of lipocytes in regenerating rat liver, J. Hepatol. 12 (1991), 344-350.

[22] A. Tannapfel, R. Kühn, H. Keßler and Ch. Wittekind, Expression of c-erbB2 oncogene product in different tumours and its standardised evaluation, Anal. Cell. Path. 10 (1996), 149160.

[23] T. Uchida and R.L. Peters, The nature and origin of proliferated bile ductules in alcoholic liver disease, Am. J. Clin. Pathol. 79 (1983), 326-333.

[24] P. Van Eyken and V.J. Desmet, Development of intrahepatic bile ducts, ductular metaplasia of hepatocytes, and cytokeratin pattern in various types of human hepatic neoplasms, in: The Role of Cell Types in Hepatocancerogenesis, A.E. Sirica, ed., CRC Press, Boca Rotan, 1992, pp. 227-263.

[25] P. Van Eyken, R. Sciot, B. von Damme, C. DeWolf-Peters and V.J. Desmet, Keratin immunohistochemistry in normal human liver. Cytokeratin pattern of hepatocytes, bile ducts and acinar gradient, Virchows Arch. 412 (1987), 63-72. 


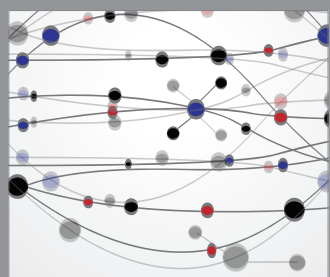

The Scientific World Journal
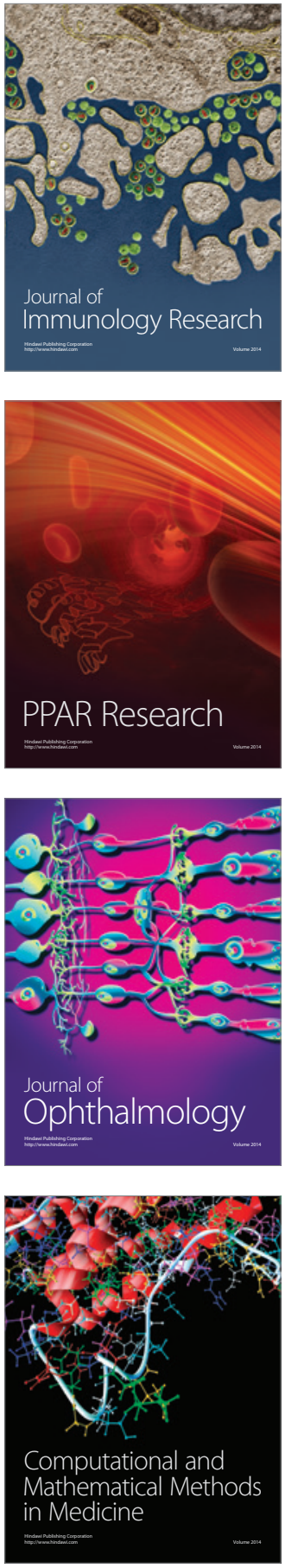

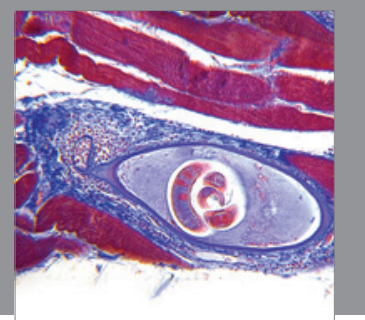

Gastroenterology

Research and Practice
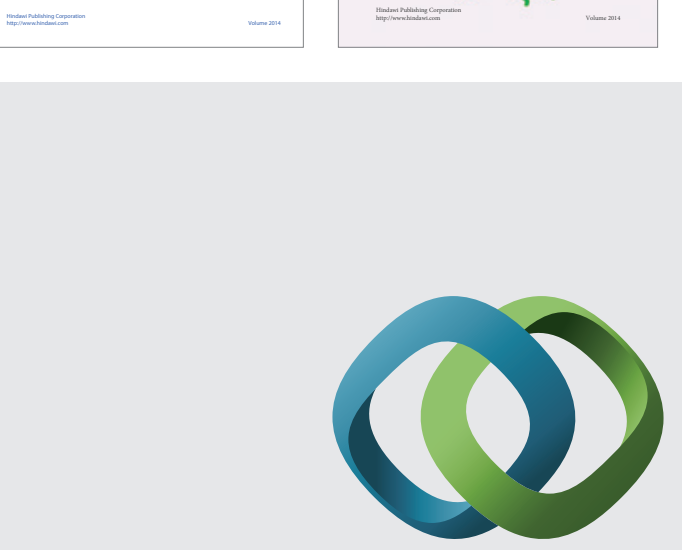

\section{Hindawi}

Submit your manuscripts at

http://www.hindawi.com
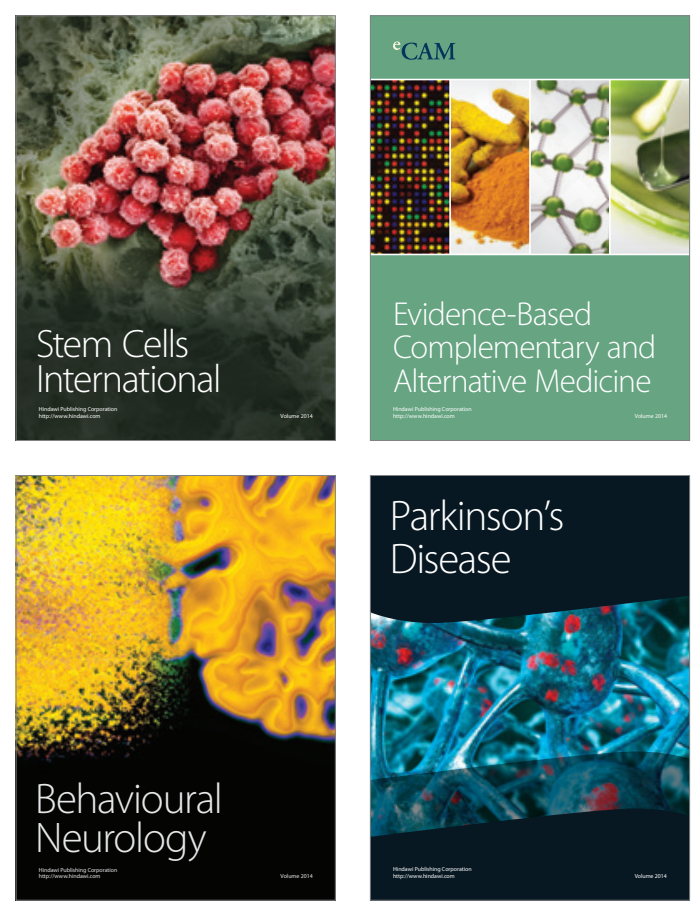

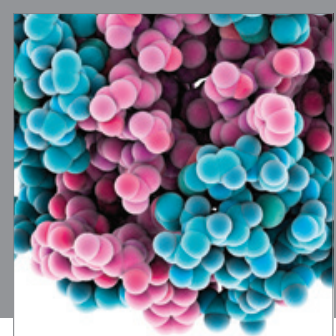

Journal of
Diabetes Research

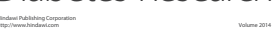

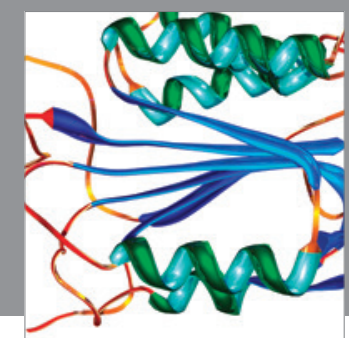

Disease Markers
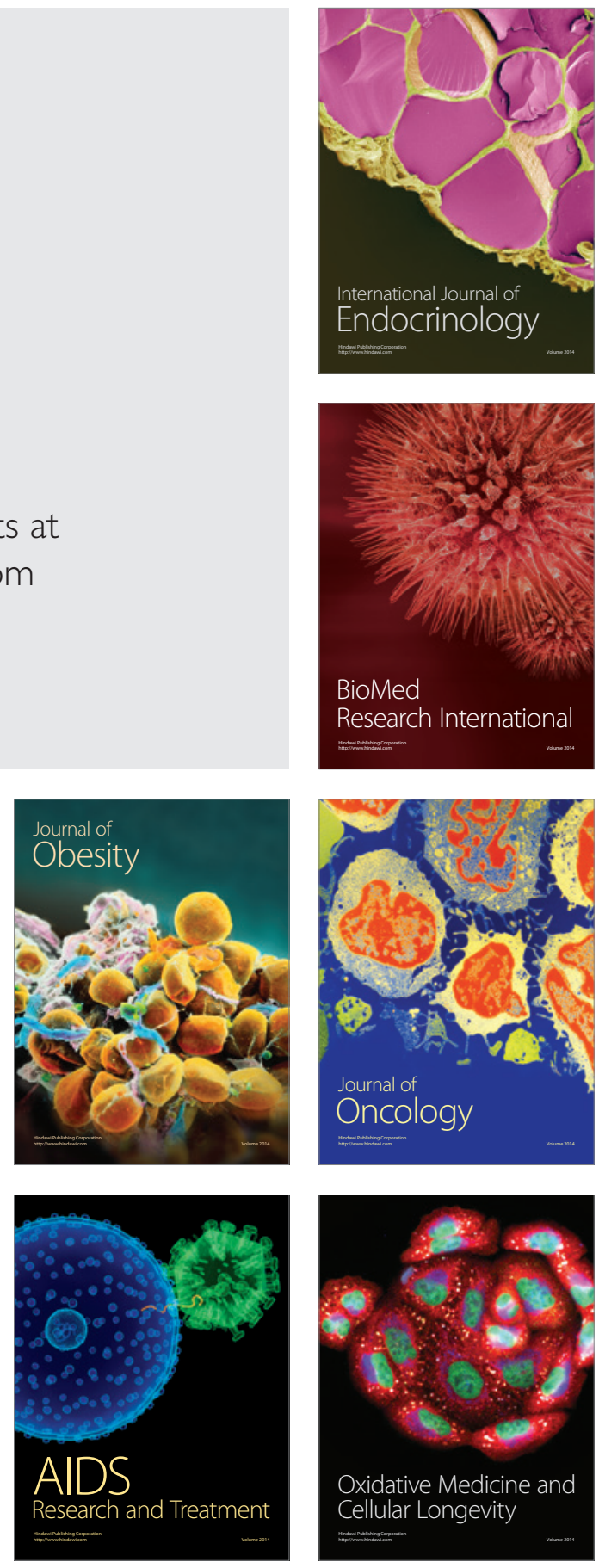